\title{
The hypothalamus-adipose axis is a key target of developmental programming by maternal nutritional manipulation
}

\section{Christophe Breton}

Unité Environnement Périnatal et Croissance, UPRES EA 4489, Equipe Dénutritions Maternelles Périnatales, Université Lille-Nord de France, Villeneuve d'Ascq, France
Correspondence should be addressed to $C$ Breton Email christophe.breton@univ-lille1.fr

\begin{abstract}
Epidemiological studies initially demonstrated that maternal undernutrition leading to low birth weight may predispose for energy balance disorders throughout life. High birth weight due to maternal obesity or diabetes, inappropriate early post-natal nutrition and rapid catch-up growth may also sensitise to increased risk of obesity. As stated by the Developmental Origin of Health and Disease concept, the perinatal perturbation of foetus/neonate nutrient supply might be a crucial determinant of individual programming of body weight set point. The hypothalamus-adipose axis plays a pivotal role in the maintenance of energy homoeostasis controlling the nutritional status and energy storage level. The perinatal period largely corresponds to the period of brain maturation, neuronal differentiation and active adipogenesis in rodents. Numerous dams and/or foetus/neonate dietary manipulation models were developed to investigate the mechanisms underlying perinatal programming in rodents. These models showed several common offspring hypothalamic consequences such as impaired neurogenesis, neuronal functionality, nuclei structural organisation and feeding circuitry hardwiring. These alterations led to a persistent reprogrammed appetite system that favoured the orexigenic pathways, leptin/insulin resistance and hyperphagia. Impaired hypothalamic sympathetic outflow to adipose tissue and/or reduced innervation may also account for modified fat cell metabolism. Thus, enhanced adipogenesis and/or lipogenesis capacities may predispose the offspring to fat accumulation. Abnormal hypothalamus-adipose axis circadian rhythms were also evidenced. This review mainly focuses on studies in rodents. It highlights hormonal and epigenetic mechanisms responsible for long-lasting programming of energy balance in the offspring. Dietary supplementation may provide a therapeutic option using a specific regimen for reversing adverse programming outcomes in humans.
\end{abstract}

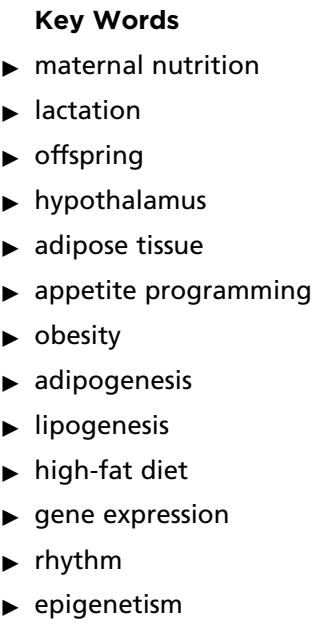

\section{Introduction}

Epidemiological studies initially demonstrated that adverse environmental factors leading to intrauterine growth retardation (IUGR) and low birth weight may predispose individuals to the later onset of development of pathologies related to the metabolic syndrome. As illustrated by the Dutch Famine Study, foetuses http://joe.endocrinology-journals.org DOI: 10.1530/JOE-12-0157
() 2013 Society for Endocrinology Printed in Great Britain
Published by Bioscientifica Ltd. 
exposed to famine during early pregnancy had a higher energy intake and adiposity in adulthood (Ravelli et al. 1999). High birth weight due to maternal obesity or diabetes, inappropriate early post-natal nutrition and more specifically rapid catch-up growth may also sensitise to increased risk of obesity. Thus, the relationship between birth weight and adult adiposity is thought of as a ' $U$ '-shaped curve with both low and high birth weight predisposing for the onset of later obesity. Originally called the Barker hypothesis or foetal programming, these observations have led to the Developmental Origin of Health and Disease (DOHaD) hypothesis (FernandezTwinn \& Ozanne 2006). This concept states that an adverse perinatal environment programmes or imprints the development of several tissues. It may then permanently determine physiological responses and ultimately produce energy balance dysfunction and diseases later in life. Thus, the perinatal perturbation of foetus/neonate nutrient supply has been proposed to be a key determinant, especially the degree of mismatch between the pre- and post-natal environments (Gluckman et al. 2008).

The hypothalamus-adipose axis highly contributes to the maintenance of energy homoeostasis controlling nutritional status and energy storage level (Fig. 1). The hypothalamus is composed of several nuclei that produce neuropeptides involved in key physiological functions. In adults, it plays a pivotal role, especially the mediobasally located arcuate nucleus (Arc), in food intake and energy homoeostasis regulation. The Arc integrates peripheral information such as hormones (insulin and ghrelin), adipocytokines (leptin) and nutrients (glucose and free fatty acid). Leptin, together with insulin, acts on its respective receptor $\mathrm{Ob}-\mathrm{Rb}$ and insulin receptor (InsR), both linked to the common phosphatidylinositol 3-kinase (PI3K) pathway, to reduce the expression and release of hypothalamic orexigenic peptides such as neuropeptide $\mathrm{Y}$ (NPY)/agouti-related peptide and activate anorexigenic peptides such as $\alpha$-melanocyte-stimulating hormone $(\alpha$-MSH, a neuropeptide derived from pro-opiomelanocortin (POMC) processing in the hypothalamus)/cocaine- and amphetamine-regulated transcript. Then, the Arc drives other hypothalamic areas such as ventromedial (VMN), dorsomedial (DMN) and paraventricular (PVN) nuclei (considered as satiety centres) and the lateral hypothalamic area (considered as hunger centre) (Arora \& Anubhuti 2006). Several hypothalamic nuclei, especially the PVN, may, in turn, modulate, via the sympathetic autonomic nervous system, energy expenditure such as lipolysis and/or thermogenesis in adipose tissue (Fliers et al. 2003; Fig. 2).

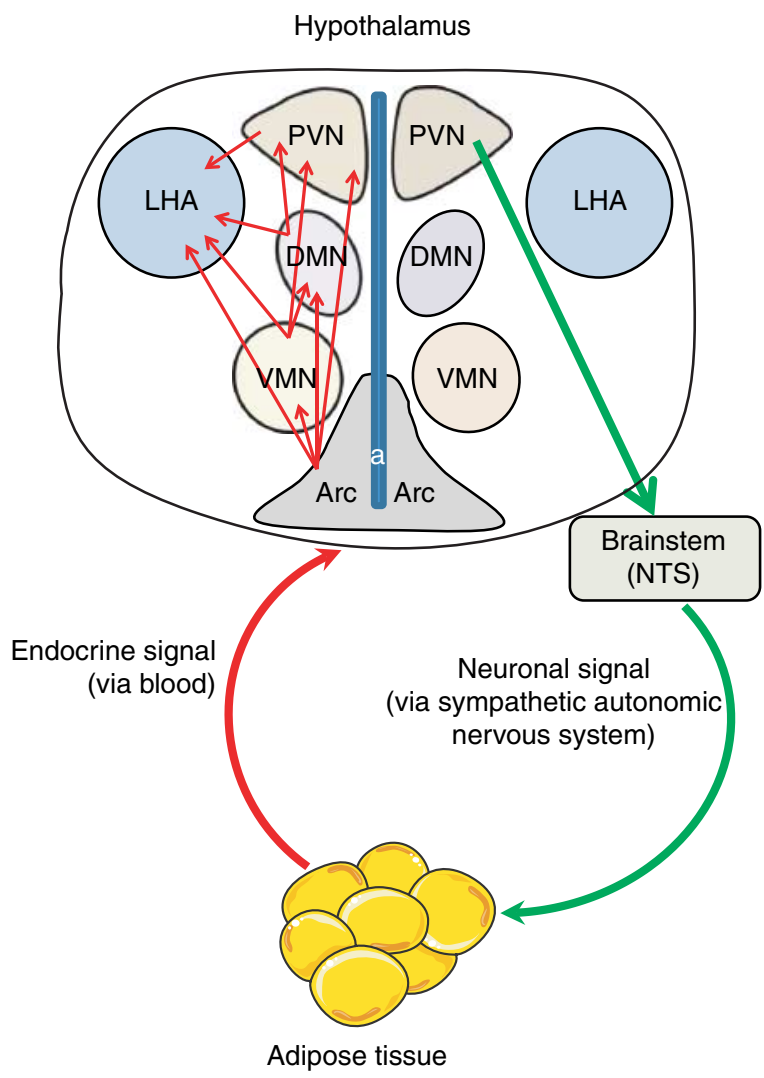

Figure 1

Schematic overview of the hypothalamus-adipose axis (based on rat data) involved in food intake regulation and energy expenditure. In short, the arcuate nucleus (Arc) integrates peripheral endocrine signal via blood (such as leptin). Leptin acts on its receptor to modulate the expression and release of Arc appetite-regulating neuropeptides. Then, the Arc drives other hypothalamic areas such as ventromedial (VMN), dorsomedial (DMN) and paraventricular (PVN) nuclei (considered as satiety centres) and the lateral hypothalamic area (LHA; considered as a hunger centre). Coronal section shows the relative position of these nuclei with respect to each other through the hypothalamus. Circuits allowing communications between these neuronal populations are indicated by red arrows. Neuronal signal, especially from the PVN, modulates via the nucleus of the solitary tract (NTS) located in the brainstem, the activity of sympathetic autonomic nervous system (indicated by a green arrow). Then, it regulates energy expenditure such as lipolysis and/or thermogenesis in adipose tissue.

The adipocyte is a specialised cell that stores excess energy as triacylglycerol (TG) in lipid droplets during lipogenesis. When energy is required, the stored TG is hydrolyzed via activation of lipolytic pathways, mainly driven by noradrenergic innervation. Both lipogenesis and lipolysis mechanisms are complicated and highly regulated cellular processes (Fig. 3). Besides its effects on adipocyte lipid mobilisation, sympathetic innervation of white adipose tissue (WAT) may act as an inhibitor of fat cell number via inhibition of fat cell proliferation

Published by Bioscientifica Ltd 
(Bowers et al. 2004). The adipocyte is also an endocrine cell, producing adipocytokines, hormones, appetiteregulating related peptides or receptors associated with the control of energy balance. On the one hand, some of these circulating factors act as adipocyte lipid metabolism regulators in an autocrine/paracrine manner. On the other hand, in addition to leptin, some of them act as peripheral endocrine signals to regulate hypothalamic energy homoeostasis (Wang et al. 2008).

To date, rodents are the most common models used to investigate the mechanisms underlying hypothalamusadipose axis developmental programming. Indeed, the perinatal period of life largely corresponds to the period of brain maturation, neuronal differentiation and active adipogenesis. The differentiation of these systems occurs primarily during the last week of gestation in the rat, accelerates during early post-natal life, still being active after weaning. During this period, hypothalamic circuitry regulating energy homoeostasis organisation as well as adipocyte precursors is still plastic and very sensitive to maternal factors, including both metabolic factors and nutritional environment (Bouret et al. 2004). The WAT expansion involves either adipogenesis (i.e. proliferation and differentiation resulting from the recruitment of new preadipocytes from progenitor cells) and/or lipogenesis leading to triglyceride accumulation in pre-existing or mature adipocytes. Among numerous transcription factors, Pparg is unique in being able to promote both mechanisms (Muhlhausler \& Smith 2009; Fig. 3).

This review aims to provide an overview of the impact of perinatal nutritional manipulation on offspring hypothalamus-adipose axis. It mainly focuses on studies in rodents. We then summarise the possible developmental programming mechanisms underlying long-lasting perturbation of this axis throughout life.

\section{Prenatal effects}

\section{Maternal reduced nutrition}

Two models have been mainly described: maternal lowprotein (LP) isocaloric diet during gestation and/or lactation and global maternal food restriction ranging from 20 to $70 \%$ of control intake.

Offspring of undernourished mothers exhibit structural disorganisation and malprogramming of the appetite-regulating system in the hypothalamus Maternal reduced nutrition modifies the hypothalamic cytoarchitectonic organisation in the offspring. Weanling rat offspring from dams fed a LP diet presented nuclei morphometric alterations such as a greater volume of the VMN and PVN associated with higher neuronal densities (Plagemann et al. 2000). Maternal nutrient restriction may also affect cell proliferation in the offspring. Weanling rat offspring from $20 \%$ food-restricted dams (FR20) during the first 12 days of pregnancy had fewer total cells and NPY and $\alpha$-MSH neurons in the Arc (García et al. 2010).

Maternal nutrient restriction alters hypothalamic appetite-regulating neuropeptide mRNA levels in the offspring, favouring the orexigenic pathways. Weanling rat offspring from LP dams displayed enhanced orexigenic drive (i.e. increased $N P Y$ and decreased POMC mRNA expression; Cripps et al. 2009). Thus, it may predispose them to hyperphagia and an increased risk of developing obesity later in life, particularly when nourished with a hypercaloric diet. Indeed, post-weaning high-fat (HF) diet exacerbated the catch-up growth of rat offspring from FR70 dams during gestation, leading to higher energy intake and adiposity. Elevated NPY mRNA expression levels were observed in adulthood (Ikenasio-Thorpe et al. 2007).

Maternal undernutrition affects the organisation of the hypothalamic feeding circuitry hardwiring in the offspring. Neonatal offspring from LP mothers (Coupé et al. 2010) and weanling pups from FR50 dams from the last week of gestation and lactation (Delahaye et al. 2008) displayed a strong reduction of $\alpha$-MSH-immunoreactive fibres innervating the PVN. In addition, fasted adult offspring from FR70 dams during gestation displayed no marked reduced $\alpha$-MSH-immunoreactive fibre projection intensity in the PVN (Breton et al. 2009).

Maternal reduced nutrition modifies circadian rhythms in the offspring. Weanling offspring rats from LP dams displayed alterations of the hypothalamic circadian expression profile of mRNA encoding clock genes. Gene expression modifications were associated with subtle modified circadian feeding pattern (OrozcoSolís et al. 2011). Adult offspring from FR70 dams during gestation also exhibited modified daily light/dark cycle feeding rhythm and refeeding time course after fasting (Breton et al. 2009). In agreement with these findings, adult mice from LP dams cross-fostered to control lactating dams had abnormal feeding circadian rhythms before the onset of obesity. They exhibited diurnal increased hypothalamic NPY mRNA expression levels with lights-on hyperactivity. Animals also presented enhanced WAT mRNA expression levels of lipogenic and clock genes coinciding with the period of maximum food consumption (Sutton et al. 2010).

Published by Bioscientifica Ltd. 


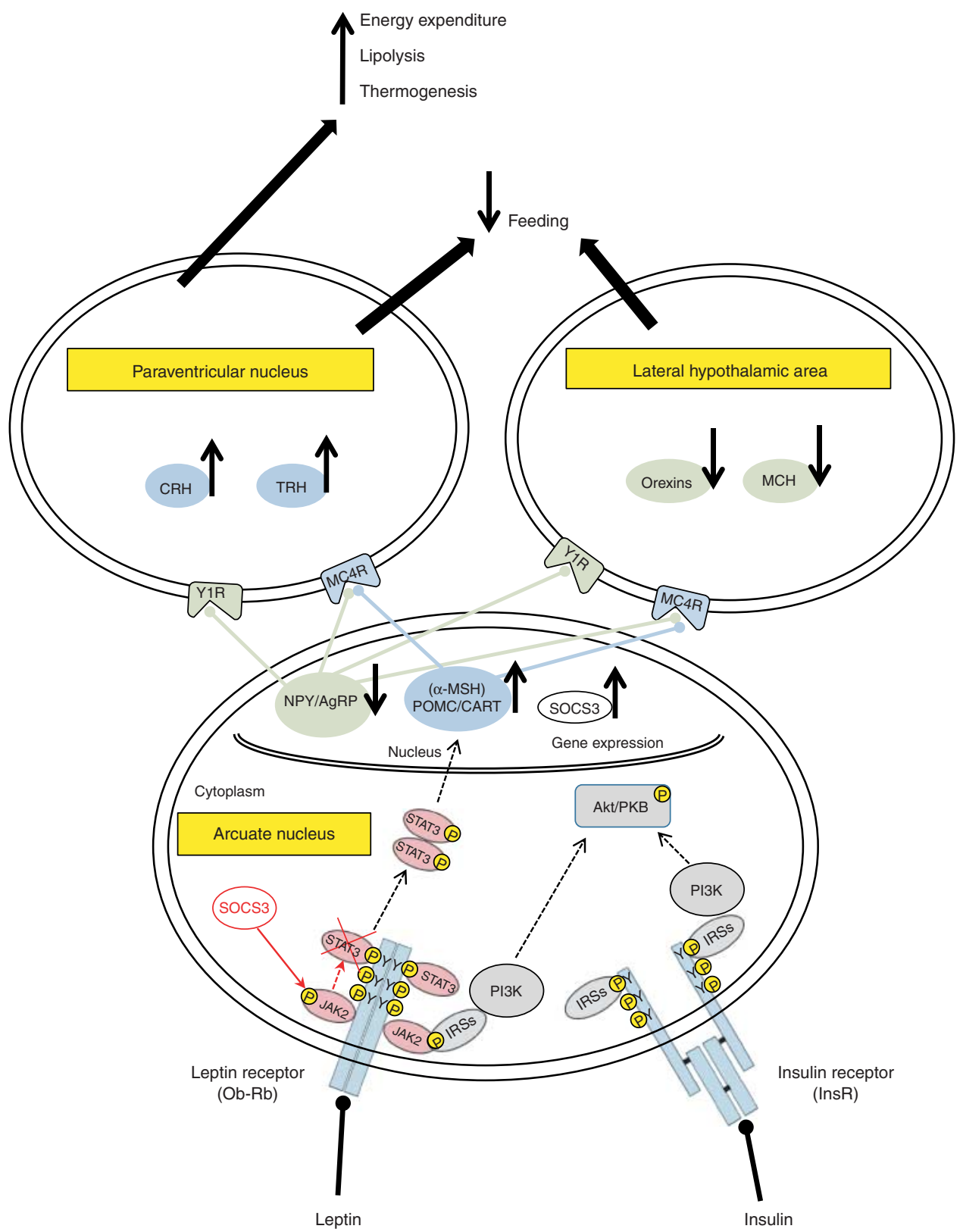

Figure 2

Schematic representation of leptin/insulin intracellular signalling pathways in the arcuate nucleus of hypothalamus. To simplify the figure, only factors that are primary targets of maternal nutrition manipulation have been represented. Leptin binding to its receptor $(\mathrm{Ob}-\mathrm{Rb})$ induces activation of JAK2, receptor dimerisation, JAK2-mediated phosphorylation of intracellular part of Ob-Rb, phosphorylation and activation of STAT3. Activated STAT3 dimerises and translocates to the nucleus to transactivate target genes. Modulation of gene expression is indicated by black arrows. Insulin binding to its receptor (InsR) induces receptor tyrosine autophosphorylation and activation of insulin receptor substrates (IRSs)/phosphatidylinositol 3-kinase (PI3K)/protein kinase B (Akt/PKB) signalling pathways. Both hormones reduce the expression and release of orexigenic peptides such as neuropeptide Y (NPY)/agouti-related peptide (AgRP) and activate anorexigenic peptides such as $\alpha$-melanocyte-stimulating hormone $(\alpha-\mathrm{MSH}$, a neuropeptide derived from pro-opiomelanocortin (POMC) processing in Printed in Great Britain the hypothalamus)/cocaine- and amphetamine-regulated transcript (CART). Leptin also activates the expression of suppressors of cytokine signalling 3 (SOCS3) that in turn inhibits leptin-induced tyrosine phosphorylation of JAK2 and STAT3 activation (indicated in red). The direct cross talk between insulin and leptin signalling at the level of JAK2/IRSs/PI3K is represented. NPY that selectively binds to Y1 NPY receptor (Y1R) induces the expression and release of orexigenic peptides (i.e. orexins and melanin concentrating hormone (MCH) in the lateral hypothalamic area) and decreases the expression and release of anorexigenic peptides (i.e. corticotropin-releasing hormone (CRH) and thyrotropin-releasing hormone (TRH) in the paraventricular nucleus), thereby increasing food intake and decreasing energy expenditure. By contrast, $\alpha$-MSH that binds to MC4 receptor (MC4R) acts on the opposite way, thereby decreasing food intake and increasing energy expenditure. 
Overall, these observations indicate that the hypothalamic organisation and appetite regulatory system as well as the hypothalamus-adipose axis circadian clock undergo long-term nutritional programming. Although the underlying mechanisms remain unclear, several studies have described the association between the disruption of the circadian clock and metabolic dysfunction (Maury et al. 2010). Thus, given that the clock genes also regulate the expression of several genes involved in adipogenesis and lipogenesis, and because of the global increase in energy intake, these observations strongly suggest that the perturbations of the hypothalamusadipose axis might increase propensity for diet-induced obesity in adulthood.

Offspring of food-restricted dams show central leptin resistance The adipocyte-derived hormone leptin and the pancreatic $\beta$-cell-derived hormone insulin each function as afferent signals to the hypothalamus in an endocrine feedback loop that regulates body adiposity. The ineffectiveness of leptin/insulin to inhibit energy intake and increase energy expenditure is termed resistance (Fig. 2).

Weanling rat offspring from FR20 dams during the first 12 days of pregnancy exhibited higher hypothalamic SOCS3 mRNA expression levels (a key negative-feedback regulator of leptin signalling involved in leptin resistance). Adult rats showed hyperphagic obesity with persistent impaired insulin/leptin sensitivity (García et al. 2010). Rat offspring from FR50 dams from day 10 to term gestation showed reduced leptin-stimulated phosphorylated STAT3 (pSTAT3) in the hypothalamus (Desai et al. 2007). The blunted phosphorylation of this key transcription factor of the leptin receptor signalling pathway highly suggests a leptin-resistant state. These mechanisms may contribute to the hypothalamic upregulation of orexigenic pathways associated with blunted activation of the sympathetic nervous system, possibly disturbing long-term body weight set point.

\section{Offspring of LP malnourished mothers show an} age-related loss of insulin sensitivity in adipocytes Adipocytes of young adult offspring from LP dams displayed modified functionality. First, they showed an improved insulin sensitivity associated with increased InsR as well as higher basal and insulin-stimulated PI3K pathway activities. Secondly, they exhibited increased noradrenergic-stimulated lipolysis and a blunted response to insulin-reduced lipolytic action. By contrast, older adult offspring from LP dams underwent an age-related loss of glucose tolerance that paralleled an impaired insulinstimulated PI3K pathway in adipocytes (Ozanne et al. 2001).

Offspring of nutrient-restricted dams are predisposed to adiposity displaying alterations in adipose tissue noradrenergic innervation and thermogenesis A large body of evidence suggests that the adipose tissue is a target of developmental programming of adult overweight by maternal undernutrition. Maternal reduced nutrition programmes an increase of fat cell size and number in the offspring. Adult rat offspring from LP dams (Guan et al. 2005) and from FR70 dams during gestation (Lukaszewski et al. 2011) showed enhanced adipogenesis (i.e. elevated PPARG activity) with impaired leptin antilipogenic action. It may be seen as an advantage to survive under poor nutrition conditions as stated by the thrifty phenotype hypothesis. In accordance with dysregulated light/dark-phase food intake rhythm, we also found that the daily transcriptional profile of several clock genes was modified in WAT offspring from FR70 dams (M A Lukaszewski and C Breton 2011, unpublished data). Post-natal catch-up growth after foetal nutrient restriction exacerbated fat accumulation leading to pronounced adipocyte hypertrophy. Adipose expression of the adipogenic and lipogenic genes was globally upregulated (Bol et al. 2009). In particular, mouse offspring from LP dams exhibited elevated adipogenic NPY system in WAT (Han et al. 2012). Overall, these results indicate that more than foetal growth retardation, the adverse effect of a rapid post-natal catch-up growth subsequent to it (due to enriched post-natal diet), might be a key determinant of programmed adiposity at adult age.

Maternal nutrient restriction modifies sympathetic activity in WAT offspring. First, weanling pups from FR50 dams from the last week of gestation and lactation exhibited elevated circulating catecholamines that could participate, via chronic $\beta$-adrenergic stimulation, in the remodelling of WAT into the thermogenically active brown adipose tissue (BAT). These phenotype changes might occur in order to increase thermogenesis, as a compensatory mechanism to overcome difficulties in maintaining their body temperature after birth (Delahaye et al. 2010). Secondly, adult rat offspring from FR50 dams from day 10 to term gestation showed a reduction in WAT noradrenergic innervation and modified adrenoreceptor subtype ratio. These mechanisms might affect adipocyte adipogenesis and/or lipolysis (García et al. 2011).

Thus, the functional impairment of the sympathetic innervation of WAT as an inhibitor of fat cell proliferation (i.e. hypercellularity) and/or activator of

Published by Bioscientifica Ltd. 
lipolysis (i.e. hypertrophy) might be a key determinant of increased adiposity in adult offspring following maternal reduced nutrition.

\section{Uterine artery ligation}

IUGR offspring are prone to develop adiposity in adulthood Uterine artery ligation in the pregnant dam reduces the blood flow to the foetuses and is used as a model for placental insufficiency. In addition to global nutrient reduction, oxygen restriction is also created by uterine artery ligation causing persistent IUGR.

Subtle alterations in NPY gene expression have been described in foetal and post-natally uteroplacental insufficiency offspring, possibly disturbing long-term body weight set point (Huizinga et al. 2001). Although adult

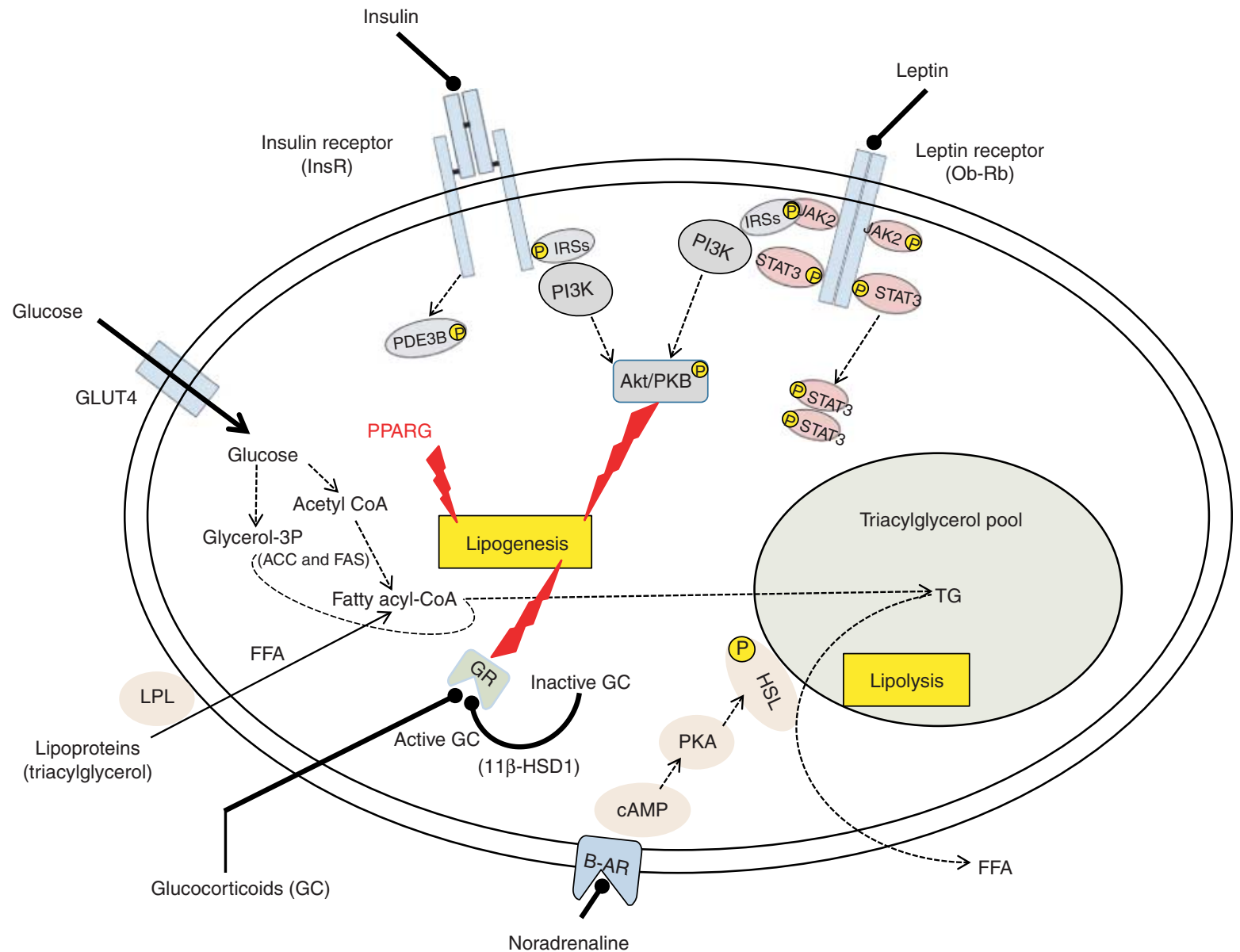

\section{Figure 3}

Schematic representation of basic steps in lipogenesis and lipolysis in the adipocyte. To simplify the figure, only mechanisms that are primary targets of maternal nutrition manipulation have been represented. Triacylglycerol (TG) circulates in blood in the form of lipoproteins. Free fatty acids (FFA) that are released from lipoproteins, catalysed by lipoprotein lipase (LPL), diffuse into the adipocyte. Intracellular FFA are converted to fatty acyl-CoA and are then re-esterified to form TG using glycerol-3 phosphate (glycerol-3P) that is generated by glucose metabolism. FFA may also originate from acetyl-CoA (de novo lipogenesis) driven by the lipogenic enzymes acetylCoA carboxylase (ACC) and fatty acid synthase (FAS). Lipolysis occurs via a CAMP-mediated cascade, which results in the phosphorylation of hormonesensitive lipase (HSL), an enzyme that hydrolyzes TG into FFA and glycerol. These FFA are then free to diffuse into the blood. Insulin enhances the storage of fat as TG by increasing LPL and lipogenic enzyme activities. It also facilitates the transport of glucose by stimulating the GLUT4 glucose transporter. In addition, phosphorylation and activation of cyclic nucleotide phosphodiesterases $3 \mathrm{~B}$ (PDE3B) is a key event in the antilipolytic action of insulin, decreasing CAMP levels in adipocytes. By contrast, leptin presents antilipogenic and lipolytic effects by suppressing expression and activity of lipogenic enzymes and PPARG. Noradrenaline released from the sympathetic autonomic nervous system binds $\beta$-adrenoreceptor ( $\beta$-AR) and activates lipolysis. Prolonged exposure to glucocorticoids (GC) that bind intracellular glucocorticoid receptor (GR) enhances adipogenesis. This may be due either to an increase in circulating GC and/or to an increase in

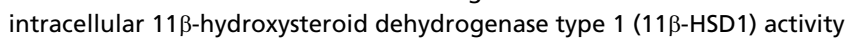
that predominantly converts inactive cortisone to active corticosterone, thus amplifying local GC action. http://joe.endocrinology-journals.org DOI: 10.1530/JOE-12-0157
(C) 2013 Society for Endocrinology Printed in Great Britain 
offspring developed marked adiposity, Pparg mRNA expression level elevation in WAT occurred before the onset of overt obesity (Joss-Moore et al. 2010).

\section{Maternal overnutrition}

Maternal overnutrition and obesity were induced by feeding the dams HF or high-energy/cafeteria (fat and sugar) diets before (periconceptional period) and/or during gestation and/or lactation.

Offspring of overfed mothers exhibit malprogramming of the appetite-regulating system in the hypothalamus Maternal overnutrition stimulates in utero hypothalamic neurogenesis in the offspring. Foetal rat of dams fed a HF diet during the last 2 weeks of gestation exhibited increased hypothalamic proliferation of orexigenic peptide-expressing neurons. Thus, persistent elevated expression and production of these peptides may increase risk for overeating and obesity later in life (Chang et al. 2008).

Maternal HF consumption permanently reprogrammes the hypothalamic appetite system to favour the orexigenic pathways in the offspring. Most studies reported that weanling rat HF-fed dams were more prone to hyperphagia and obesity. In particular, rat offspring from HF-fed mothers before mating and throughout gestation showed reduced hypothalamic anorexigenic pathways (i.e. POMC) whereas orexigenic NPY Y1 receptor expression levels were increased (Rajia et al. 2010). In adulthood, these animals still displayed marked hyperphagia with longlasting increased orexigenic pathways (i.e. NPY activation and POMC inhibition). Post-natal overnutrition of offspring from obese dams amplified these hypothalamic changes (Morris \& Chen 2009). Finally, rats born to cafeteria diet-fed mothers displayed a greater preference for the same range of foodstuffs (Bayol et al. 2008).

\section{Offspring born to overfed mothers show central} leptin resistance Besides their increased orexigenic pathways, additional findings suggest that offspring from obese/overfed dams exhibit early and persistent central leptin resistance. First, both pre- and post-natal maternal overnutrition programmed higher hypothalamic SOCS3 mRNA expression levels with blunted leptin-induced pSTAT3 in the offspring (Rajia et al. 2010). Secondly, post-weaning overnutrition potentiated a maternal dietinduced increase in hypothalamic SOCS3 mRNA expression levels and impairment of the PI3K pathway (Morris \& Chen 2009).
Offspring of obese dams are predisposed to adiposity Several studies demonstrate that maternal body composition at conception and during gestation and lactation has important implications for offspring adiposity. In rodents, offspring from obese dams fed cafeteria diet before mating and/or throughout gestation and lactation were overweight, displaying marked adiposity. The adipocyte hypertrophy was accompanied by reduced lipolytic adrenoreceptors as well as raised adipogenic factor mRNA expression levels such as 11ß-HSD1 (that catalyses the interconversion of inactive 11-dehydrocorticosterone to active corticosterone) and Pparg. This profile was exacerbated under a HF diet (Samuelsson et al. 2008). They also had abnormalities of WAT fatty acid composition (Bayol et al. 2008).

Overall, although offspring from obese dams gain more fat mass than those from control dams independent of lactation and/or post-weaning diet, overnutrition during these periods always worsens adipogenesis programming. These observations highlight the importance of post-natal diet in modifying adiposity.

Taken together, as observed in maternal reduced nutrition models, these programmed mechanisms might contribute directly and/or indirectly toward irreversible long-term higher energy intake and impaired fat storage in adipocytes.

\section{Maternal diabetes}

In rodents, gestational diabetes (GD) is mostly induced by pancreatic islet toxin streptozotocin (STZ) injections in early pregnancy leading to maternal insulin deficiency (with further hyperglycaemia). This model is not equivalent to human GD, which is characterised by insulin resistance.

\section{Offspring from diabetic dams exhibit hypo-} thalamic appetite-regulating system dysfunctions and increased adiposity Rat GD offspring presented an increase in hypothalamic insulin levels. They showed structural and hypothalamic appetite system malprogramming. They had decreased neuron cellularity within the Arc and VMN. Adult rat GD offspring were overweight and hyperphagic with an elevated number of orexigenic NPY neurons in the Arc (Plagemann et al. 1999a). By contrast, adult mouse GD offspring displayed a persistent reduction in $\alpha$-MSH fibre densities in the PVN with altered leptin sensitivity. They also exhibited adipocyte enlargement (Steculorum \& Bouret 2011). Overall, disparity between related models points out that

Published by Bioscientifica Ltd 
the difference of species (rat vs mouse) and the timing of the STZ exposure are critical determinants in hypothalamic programming outcomes.

The impact of maternal GD exclusively during the suckling period has been investigated by cross-fostering pups of control rat dams to GD mothers. At weaning, cross-fostered offspring showed increased NPY neuron immunoreactivities. They also exhibited an increased total number of neurons in the PVN whereas no morphometric modifications were observed in the VMN (Fahrenkrog et al. 2004).

Overall, these studies indicate that exposure to a diabetic intrauterine milieu (i.e. increased insulin levels during development) leads to malorganisation of the VMN in GD offspring whereas exclusive exposure to milk from diabetic dams rather results in neural alterations in the PVN. Both protocols lead to persistent enhanced hypothalamic orexigenic drive and adiposity.

\section{Post-natal effects}

\section{Altered feeding in the neonatal period}

A few models have been developed to precisely delineate the impact of altered feeding in neonates, showing the importance of energy intake during the lactation period in predisposing offspring to adiposity.

Short lactation length leads to adiposity in the offspring Early weaning induced by interrupting maternal lactation on day 18 led to weanling rat offspring that had lower body weight. After catch-up growth, adult offspring developed hypothalamic leptin resistance. They also exhibited hyperphagia, higher body weight and adiposity (Bonomo et al. 2007). These models reinforce the notion that shortening of the lactation period may account for metabolic programming of pathologies. However, the programming effect of milk suppression depends on the developmental stage of offspring.

Modification of milk composition causes hyperphagia and obesity in the offspring Rat offspring artificially raised by gastrostomy ('pup in the cup' model) under an isocaloric high-carbohydrate milk formula (HC) (in contrast to rat milk wherein the major source of calories is fat) exhibited persistent hyperphagia and adiposity. HC rat offspring globally favoured the hypothalamic orexigenic pathways (while leptin/insulin pathways were impaired; these findings suggest a state of leptin insulin resistance). They also exhibited enhanced lipogenic enzyme activities within WAT (Srinivasan et al. 2008). Thus, as observed in offspring of diabetic dams, the altered hormonal environment of neonatal HC rats (i.e. increased insulin levels) could be an important cue for the development of the predisposition for adult-onset obesity.

\section{Energy intake during lactation programmes hypothalamic appetite-regulating systems and adiposity in the offspring Offspring of overfed} dams only throughout lactation are sensitised to adiposity. First, adult rat offspring exposed to maternal cafeteria diet during lactation exhibited higher WAT mass (Wright et al. 2011). Secondly, high-fructose diet intake by lactating dams induced hyperphagia and overweight in adult rat offspring. They showed reduced hypothalamic anorexigenic pathways as well as impaired leptin-stimulated pSTAT3 suggesting a leptin-resistant state. They exhibited enlarged adipocytes in WAT (Alzamendi et al. 2010).

By contrast, offspring of food-restricted dams only throughout lactation become less prone to diet-induced obesity. Rat offspring from FR30 dams only during the suckling period were hypophagic and displayed persistent lower body weight with adipocyte atrophy. Adult offspring exhibited beneficial gene expression programming (i.e. activated anorexigenic pathways in the hypothalamus and decreased expression of adipogenic genes in WAT). HF-fed offspring also displayed a better adaptation to the HF diet that might protect against obesity (Palou et al. 2011). Overall, these findings fit with the notion that promoting catch-up growth in low birth weight offspring may not be beneficial for their long-term outcome.

\section{Litter size modification model}

The fact that, unlike humans, most of the developmental processes of the hypothalamus-adipose axis take place after birth in rodents might explain why post-natal dietary manipulations of the lactating pups have been extensively used. Birth weight was not affected and either post-natal over- (small litters (SLs)) or undernutrition (large litters (LLs)) can be achieved immediately after birth to rapidly modify milk intake and, thus, post-natal growth and body weight until weaning.

Offspring reared in SL display a reprogrammed hypothalamic appetite-regulating system and leptin resistance Neonatally overfed SL offspring showed a long-lasting hyperphagic obese phenotype. Weanling SL rats presented increased hypothalamic insulin levels and

Published by Bioscientifica Ltd. 
persistent activation of the orexigenic pathways. First, they displayed neuronal morphometric modifications and altered hypothalamic nuclei activity (Plagemann et al. 1999b). Secondly, they exhibited impaired Arc neuron sensitivity, especially to leptin and insulin with higher NPY mRNA expression levels (López et al. 2005).

Offspring reared in SL are predisposed to adiposity showing programmed adipose tissue sympathetic activity, thermogenesis and glucocorticoid metabolism Adult SL offspring developed persistent higher fat mass. Hypertrophied adipocytes displayed global enhanced lipogenic activity accompanied by an induction of glucocorticoid (GC) receptor and 11ß-HSD1 mRNA expression levels. HF-fed SL offspring exacerbated this profile (Boullu-Ciocca et al. 2008). These observations emphasise the pivotal role of the GC WAT environment during the perinatal period on the subsequent development of obesity. In addition, adult SL rats exhibited a reduced BAT thermogenesis, modified lipolytic adrenoreceptor subtypes ratio and impaired sympathetic outflow activity that might affect lipolysis (Xiao et al. 2007).

Thus, as observed in maternal reduced nutrition models, it is tempting to speculate that the predisposity to adiposity in the offspring are attributable, at least in part, to the impairment in sympathetic activity in adipose tissue. It could presumably occur through a reprogramming of the hypothalamic metabolic circuitry and alteration in the hypothalamic control of sympathetic outflow.

\section{Offspring reared in LLs have a lower susceptibility} to diet-induced obesity Neonatally underfed LL offspring presented persistent lower body weight and adiposity. Surprisingly, weanling LL offspring also exhibited hypothalamic increased orexigenic drive (i.e. elevated NPY) with higher energy intake (Plagemann et al. 1999b). These parameters were normalised in older animals. In addition, neonatal LL offspring from DIO rat dams presumably increased leptin sensitivity and protected them from becoming obese (Patterson et al. 2010). As described in offspring from undernourished dams during lactation, reduced energy intake during the suckling period may have a long-term protective effect against obesity.

\section{Programming mechanisms}

The offspring phenotype observed after maternal nutritional manipulation depends on two closely interacting parameters. First, multiple and complex genetic factors may sensitise individuals to energy balance dysfunction, as demonstrated by studies using opposite rat polygenic substrain models (i.e. DIO vs diet resistant; Patterson et al. 2010). Secondly, environmental conditions during neonate development such as nutritional perturbation of circulating factor levels, other than nutrients, may also account for long-lasting programming of energy balance.

\section{Circulating factors}

In rodents, hormonal factors have received increasing attention over the past 10 years. In particular, inappropriate leptin levels might be a key factor of perinatal programming. First, leptin was found to act as a neurotrophic factor by promoting neuronal outgrowth from the Arc to the PVN during the early post-natal period. It is thus highly involved in the plasticity and hardwiring of the hypothalamic appetite regulatory circuits (Bouret et al. 2004). Secondly, perinatal leptin manipulation had long-term effects on offspring hypothalamus-adipose axis regulation (Granado et al. 2012). Thirdly, post-natal leptin surge was modified following maternal nutrition manipulation (i.e. suppressed, Delahaye et al. (2008)). Finally, leptin activated adipogenesis by promoting differentiation of preadipocytes (Bol et al. 2009) whereas it showed antilipogenic effects on mature adipocytes (Huan et al. 2003).

Increased insulin levels might also be a key factor of perinatal programming. First, adult rat offspring from gestational dams injected with insulin exhibited enhanced neurite outgrowth of noradrenergic fibres in the PVN (Jones et al. 1996). Secondly, post-natally intrahypothalamically insulin-treated rats that became overweight in adulthood exhibited hypotrophic neuronal nuclei within the VMN and DMN (Plagemann et al. 1999c). Finally, insulin was a critical regulator of adipogenesis whereas it promoted lipogenesis and inhibited lipolysis in mature adipocytes (Poulos et al. 2010).

Increased GC levels could be a potent factor of perinatal programming. First, corticosterone regulates synaptic organisation of POMC and NPY neurons in adult mice (Gyengesi et al. 2010). Secondly, maternal nutritional manipulation coincided with elevated perinatal circulating concentrations of GC. It caused permanent disturbed HPA axis feedback in the offspring that may contribute to obesity in adulthood (Lesage et al. 2006). Thirdly, long-term programming effects on the offspring's hypothalamusadipose axis following synthetic GC injection in pregnant dams have been largely described (Harris \& Seckl 2011). Finally, GC activated adipogenesis by promoting differentiation of preadipocytes whereas it decreased lipolysis on mature adipocytes (Poulos et al. 2010).

Published by Bioscientifica Ltd. 
Other factors (i.e. insulin-like growth factor 1 , ghrelin, neurotrophins, thyroid hormones, prolactin, inflammatory cytokines and catecholamines) might be valuable candidates.

\section{Epigenetic mechanisms}

Maternal nutritional manipulation is now considered as a transient environmental challenge that may permanently imprint the offspring genome and sensitise offspring to metabolic syndrome in adulthood. Indeed, perinatal food manipulation causes epigenetic alterations by modifying methylation of gene promoter regions (i.e. the CpG sites) and/or chromatin histone acetylation and/or miRNA expression in the offspring. These nutritionally epigenetic mechanisms may persistently affect fine tuning transcription of key genes involved in energy balance, thus programming a long-term dysfunctional hypothalamus-adipose axis.

In rodents, the hypothalamic POMC gene promoter region is a key target of epigenetic changes following perinatal nutritional manipulation. The POMC promoter was found to be less methylated in weanling rat offspring from LP dams (Coupé et al. 2010) whereas it showed hypermethylation in weanling SL animals. In the latter case, these modifications might impair hormonal effects on POMC expression and might account for hypothalamic leptin/insulin resistance (Plagemann et al. 2009). This was a very specific effect in that no change was observed in the NPY promoter region, despite increased NPY mRNA expression levels (López et al. 2005).

In WAT, adult mice from LP dams presented hypomethylation of the leptin promoter. Juvenile rat offspring from LP mothers also exhibited an increase in miRNA-483-3p mRNA expression levels encoding a protein that regulates later adipogenesis stages (FerlandMcCollough et al. 2012).

\section{Extrapolation across mammalian species}

The question arises as to whether extrapolating rodent data across higher mammalian are really relevant. Indeed, the timing of the hypothalamus-adipose axis development and, therefore, the window of vulnerability to an environmental insult differ markedly among species. In rodents that are born developmentally immature, hypothalamic neuroendocrine maturation as well as adipogenesis mainly occurs during the post-natal period. By contrast, these phenomena essentially take place before birth in bigger mammals such as sheep or primates
(Muhlhausler \& Smith 2009). In addition, genetic background-, diet-, age-, gender-, and adipose tissue depotspecific developmental programming already occur among rodent species. Then, differences might be even more important between rodents and higher species.

However, several studies clearly showed that altricial and precocial species share some similar developmental programming mechanisms. In sheep, maternal nutritional manipulation programmed the post-natal hypothalamic appetite-regulating system in the offspring (Muhlhausler et al. 2006). It was associated with changes in promoter methylation of the hypothalamic POMC gene (Stevens et al. 2010). In addition, maternal nutritional manipulation

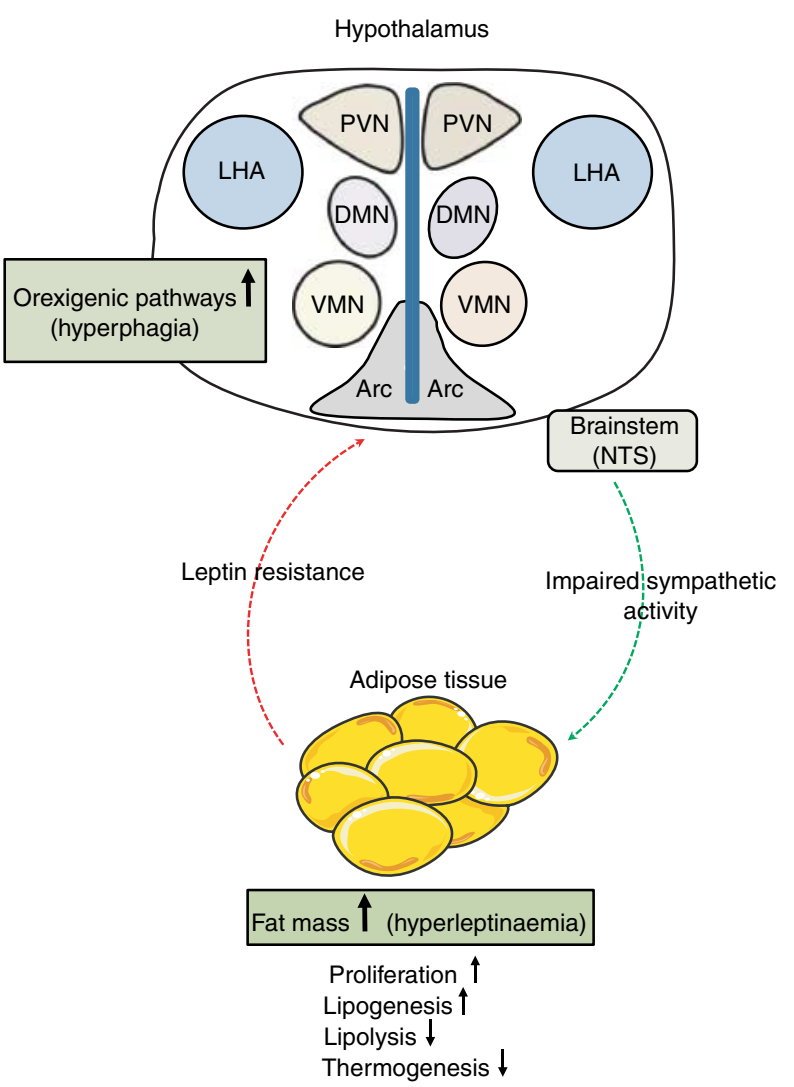

Figure 4

Schematic overview of the long-lasting effects of maternal nutritional manipulation on the hypothalamus-adipose tissue axis in the offspring. In most cases, animals display hyperleptinaemia, hypothalamic leptin resistance, impaired leptin receptor signalling pathways and enhanced orexigenic pathways (especially NPY) leading to hyperphagia. Offspring also show reduced sympathetic autonomic nervous system innervation, impaired sympathetic outflow activity and modified ratio of adrenoreceptor subtypes. These may affect adipose tissue functions (i.e. higher fat cell proliferation and lipogenesis as well as lower lipolysis and thermogenesis) sensitising to fat mass accumulation. Overall, higher energy intake, altered sympathetic activity and global increased adipogenesis and/or lipogenesis capacities may promote offspring obesity.

Published by Bioscientifica Ltd. 
also programmed fat deposition as well as adipogenic factor mRNA expression levels in WAT offspring (i.e. Pparg, Muhlhausler \& Smith (2009). In primates, third-trimester foetuses from HF-fed macaque dams also exhibited programmed hypothalamic appetite-regulating system, predisposing adult offspring to adiposity (Grayson et al. 2010).

\section{Concluding remarks}

As overviewed, gestation/lactation is a particularly sensitive period for developmental programming. In particular, endocrine and neuronal signals regulating the hypothalamus-adipose tissue axis show long-lasting perturbations in offspring (Fig. 4). In most cases, circulating leptin levels are increased. Animals display hypothalamic leptin resistance and enhanced orexigenic pathways (especially NPY) leading to hyperphagia. Offspring also show reduced sympathetic innervation and/or impaired sympathetic outflow activity that may affect adipose tissue functions. Interestingly, maternal malnutrition leads to elevated circulating NPY levels (whose origin was unclear) and an activated adipogenic NPY system in adipose tissue. Overall, higher energy intake, altered sympathetic activity and global increased adipogenesis and/or lipogenesis capacities may promote offspring obesity.

\section{Potential future areas of research}

To date, we are still far from having achieved the discovery of circulating factors as well as understanding the molecular mechanisms by which perinatal adverse nutritional conditions of the foetus/neonate sensitise to increased risk of obesity later in life. In particular, two questions remain to be answered: i) how modified circulating factor levels might be translated to the genomic level and ii) how transmission of the altered epigenetic modifications might extend beyond the F1 generation.

An increasing number of studies showed that dietary maternal supplementation (i.e. taurine, glycine, vitamin D and n-3 fatty acid) may alleviate adverse consequences of perinatal programming. In particular, folic acid (known as a methyl donor) appears to be a valuable candidate (Burdge et al. 2009). Thus, in the future, a better knowledge of the epigenomes in response to maternal developmental malnutrition raises the exciting possibility that dietary supplementation may provide a therapeutic option using a specific regimen for reversing adverse programming outcomes in humans.
Declaration of interest

The author declares that there is no conflict of interest that could be perceived as prejudicing the impartiality of the research reported.

\section{Funding}

This study was supported by grants from the French Ministry of Education and grants of the Conseil Régional du Nord-Pas de Calais.

\section{References}

Alzamendi A, Castrogiovanni D, Gaillard RC, Spinedi E \& Giovambattista A 2010 Increased male offspring's risk of metabolic-neuroendocrine dysfunction and overweight after fructose-rich diet intake by the lactating mother. Endocrinology 151 4214-4223. (doi:10.1210/en. 2009-1353)

Arora S \& Anubhuti 2006 Role of neuropeptides in appetite regulation and obesity. Neuropeptides 40 375-401. (doi:10.1016/j.npep.2006.07.001)

Bayol SA, Simbi BH, Bertrand JA \& Stickland NC 2008 Offspring from mothers fed a 'junk food' diet in pregnancy and lactation exhibit exacerbated adiposity that is more pronounced in females. Journal of Physiology 586 3219-3230. (doi:10.1113/jphysiol.2008.153817)

Bol VV, Delattre AI, Reusens B, Raes M \& Remacle C 2009 Forced catch-up growth after fetal protein restriction alters the adipose tissue gene expression program leading to obesity in adult mice. American Journal of Physiology. Regulatory, Integrative and Comparative Physiology 2 R291-R299. (doi:10.1152/ajpregu.90497.2008)

Bonomo IT, Lisboa PC, Pereira AR, Passos MC \& de Moura EG 2007 Prolactin inhibition in dams during lactation programs for overweight and leptin resistance in adult offspring. Journal of Endocrinology 192 339-344. (doi:10.1677/joe.1.06952)

Boullu-Ciocca S, Achard V, Tassistro V, Dutour A \& Grino M 2008 Postnatal programming of glucocorticoid metabolism in rats modulates high-fat diet-induced regulation of visceral adipose tissue glucocorticoid exposure and sensitivity and adiponectin and proinflammatory adipokines gene expression in adulthood. Diabetes 57 669-677. (doi:10.2337/db07-1316)

Bouret SG, Draper SJ \& Simerly RB 2004 Trophic action of leptin on hypothalamic neurons that regulate feeding. Science 304 108-110. (doi:10.1126/science.1095004)

Bowers RR, Festuccia WT, Song CK, Shi H, Migliorini RH \& Bartness TJ 2004 Sympathetic innervation of white adipose tissue and its regulation of fat cell number. American Journal of Physiology. Regulatory, Integrative and Comparative Physiology 286 R1167-R1175. (doi:10.1152/ajpregu. 00558.2003)

Breton C, Lukaszewski MA, Risold PY, Enache M, Guillemot J, Rivière G, Delahaye F, Lesage J, Dutriez-Casteloot I, Laborie C et al. 2009 Maternal prenatal undernutrition alters the response of POMC neurons to energy status variation in adult male rat offspring. American Journal of Physiology. Endocrinology and Metabolism 3 E462-E472. (doi:10.1152/ ajpendo.90740.2008)

Burdge GC, Lillycrop KA, Phillips ES, Slater-Jefferies JL, Jackson AA \& Hanson MA 2009 Folic acid supplementation during the juvenilepubertal period in rats modifies the phenotype and epigenotype induced by prenatal nutrition. Journal of Nutrition 139 1054-1060. (doi:10.3945/jn.109.104653)

Chang GQ, Gaysinskaya V, Karatayev O \& Leibowitz SF 2008 Maternal high-fat diet and fetal programming: increased proliferation of hypothalamic peptide-producing neurons that increase risk for overeating and obesity. Journal of Neuroscience 28 12107-12119. (doi:10.1523/JNEUROSCI.2642-08.2008) 
Coupé B, Amarger V, Grit I, Benani A \& Parnet P 2010 Nutritional programming affects hypothalamic organization and early response to leptin. Endocrinology 2 702-713. (doi:10.1210/en.2009-0893)

Cripps RL, Martin-Gronert MS, Archer ZA, Hales CN, Mercer JG \& Ozanne SE 2009 Programming of hypothalamic neuropeptide gene expression in rats by maternal dietary protein content during pregnancy and lactation. Clinical Science 117 85-93. (doi:10.1042/ CS20080393)

Delahaye F, Breton C, Risold PY, Enache M, Dutriez-Casteloot I, Laborie C, Lesage J \& Vieau D 2008 Maternal perinatal undernutrition drastically reduces postnatal leptin surge and affects the development of arcuate nucleus POMC neurons in neonatal male rat pups. Endocrinology 149 470-475. (doi:10.1210/en.2007-1263)

Delahaye F, Lukaszewski MA, Wattez JS, Cisse O, Dutriez-Casteloot I, Fajardy I, Montel V, Dickes-Coopman A, Laborie C, Lesage J et al. 2010 Maternal perinatal undernutrition programs a "brown-like" phenotype of gonadal white fat in male rat at weaning. American Journal of Physiology. Regulatory, Integrative and Comparative Physiology $\mathbf{1}$ R101-R110. (doi:10.1152/ajpregu.00604.2009)

Desai M, Gayle D, Han G \& Ross MG 2007 Programmed hyperphagia due to reduced anorexigenic mechanisms in intrauterine growth-restricted offspring. Reproductive Sciences 4 329-337. (doi:10.1177/ 1933719107303983)

Fahrenkrog S, Harder T, Stolaczyk E, Melchior K, Franke K, Dudenhausen JW \& Plagemann A 2004 Cross-fostering to diabetic rat dams affects early development of mediobasal hypothalamic nuclei regulating food intake, body weight, and metabolism. Journal of Nutrition 3 648-654.

Ferland-McCollough D, Fernandez-Twinn DS, Cannell IG, David H, Warner M, Vaag AA, Bork-Jensen J, Brøns C, Gant TW, Willis AE et al. 2012 Programming of adipose tissue miR-483-3p and GDF-3 expression by maternal diet in type 2 diabetes. Cell Death and Differentiation 19 1003-1012. (doi:10.1038/cdd.2011.183)

Fernandez-Twinn DS \& Ozanne SE 2006 Mechanisms by which poor early growth programs type- 2 diabetes, obesity and the metabolic syndrome. Physiology \& Behavior 88 234-243. (doi:10.1016/j.physbeh. 2006.05.039)

Fliers E, Kreier F, Voshol PJ, Havekes LM, Sauerwein HP, Kalsbeek A, Buijs RM \& Romijn JA 2003 White adipose tissue: getting nervous. Journal of Neuroendocrinology 11 1005-1110. (doi:10.1046/j.1365-2826. 2003.01096.x)

García AP, Palou M, Priego T, Sanchez J, Palou A \& Pico C 2010 Moderate caloric restriction during gestation results in lower arcuate nucleus NPY- and $\alpha \mathrm{MSH}-n e u r o n s$ and impairs hypothalamic response to fed/fasting conditions in weaned rats. Diabetes, Obesity \& Metabolism 12 403-413. (doi:10.1111/j.1463-1326.2009.01174.x)

García AP, Palou M, Sánchez J, Priego T, Palou A \& Picó C 2011 Moderate caloric restriction during gestation in rats alters adipose tissue sympathetic innervation and later adiposity in offspring. PLOS ONE 6 e17313. (doi:10.1371/journal.pone.0017313)

Gluckman PD, Hanson MA, Beedle AS \& Spencer HG 2008 Predictive adaptive responses in perspective. Trends in Endocrinology and Metabolism 19 109-110. (doi:10.1016/j.tem.2008.02.002)

Granado M, Fuente-Martín E, García-Cáceres C, Argente J \& Chowen JA 2012 Leptin in early life: a key factor for the development of the adult metabolic profile. Obesity Facts 5 138-150. (doi:10.1159/000336967)

Grayson BE, Levasseur PR, Williams SM, Smith MS, Marks DL \& Grove KL 2010 Changes in melanocortin expression and inflammatory pathways in fetal offspring of nonhuman primates fed a high-fat diet. Endocrinology 151 1622-1632. (doi:10.1210/en.2009-1019)

Guan H, Arany E, van Beek JP, Chamson-Reig A, Thyssen S, Hill DJ \& Yang K 2005 Adipose tissue gene expression profiling reveals distinct molecular pathways that define visceral adiposity in offspring of maternal protein-restricted rats. American Journal of Physiology. Endocrinology and Metabolism 4 E663-E673. (doi:10.1152/ajpendo. 00461.2004)
Gyengesi E, Liu ZW, D'Agostino G, Gan G, Horvath TL, Gao XB \& Diano S 2010 Corticosterone regulates synaptic input organization of POMC and NPY/AgRP neurons in adult mice. Endocrinology 151 5395-5402. (doi:10.1210/en.2010-0681)

Han R, Li A, Li L, Kitlinska JB \& Zukowska Z 2012 Maternal low-protein diet up-regulates the neuropeptide $\mathrm{Y}$ system in visceral fat and leads to abdominal obesity and glucose intolerance in a sex- and time-specific manner. FASEB Journal 26 3528-3536. (doi:10.1096/fj.12-203943)

Harris A \& Seckl J 2011 Glucocorticoids, prenatal stress and the programming of disease. Hormones and Behavior 59 279-289. (doi:10.1016/j.yhbeh.2010.06.007)

Huan JN, Li J, Han Y, Chen K, Wu N \& Zhao AZ 2003 Adipocyte-selective reduction of the leptin receptors induced by antisense RNA leads to increased adiposity, dyslipidemia, and insulin resistance. Journal of Biological Chemistry 278 45638-45650. (doi:10.1074/jbc.M304165200)

Huizinga CT, Oudejans CB \& Delemarre-Van de Waal HA 2001 Decreased galanin mRNA levels in growth hormone-releasing hormone neurons after perinatally induced growth retardation. Journal of Endocrinology 170 521-528. (doi:10.1677/joe.0.1700521)

Ikenasio-Thorpe BA, Breier BH, Vickers MH \& Fraser MJ 2007 Prenatal influences on susceptibility to diet-induced obesity are mediated by altered neuroendocrine gene expression. Journal of Endocrinology $\mathbf{1}$ 31-37. (doi:10.1677/joe.1.07017)

Jones AP, Olster DH \& States B 1996 Maternal insulin manipulations in rats organize body weight and noradrenergic innervation of the hypothalamus in gonadally intact male offspring. Developmental Brain Research 97 16-21. (doi:10.1016/S0165-3806(96)00128-9)

Joss-Moore LA, Wang Y, Campbell MS, Moore B, Yu X, Callaway CW, McKnight RA, Desai M, Moyer-Mileur LJ \& Lane RH 2010 Uteroplacental insufficiency increases visceral adiposity and visceral adipose PPAR $\gamma 2$ expression in male rat offspring prior to the onset of obesity. Early Human Development 86 179-185. (doi:10.1016/j.earlhumdev. 2010.02.006)

Lesage J, Sebaai N, Leonhardt M, Dutriez-Casteloot I, Breton C, Deloof S \& Vieau D 2006 Perinatal maternal undernutrition programs the offspring hypothalamo-pituitary-adrenal (HPA) axis. Stress 4 183-198. (doi:10.1080/10253890601056192)

López M, Seoane LM, Tovar S, García MC, Nogueiras R, Diéguez C \& Señarís RM 2005 A possible role of neuropeptide $Y$, agouti-related protein and leptin receptor isoforms in hypothalamic programming by perinatal feeding in the rat. Diabetologia 48 140-148. (doi:10.1007/ s00125-004-1596-z)

Lukaszewski MA, Mayeur S, Fajardy I, Delahaye F, Dutriez-Casteloot I, Montel V, Dickes-Coopman A, Laborie C, Lesage J, Vieau D et al. 2011 Maternal prenatal undernutrition programs adipose tissue gene expression in adult male rat offspring under high-fat diet. American Journal of Physiology. Endocrinology and Metabolism 3 E548-E559. (doi:10.1152/ajpendo.00011.2011)

Maury E, Ramsey KM \& Bass J 2010 Circadian rhythms and metabolic syndrome: from experimental genetics to human disease. Circulation Research 106 447-462. (doi:10.1161/CIRCRESAHA.109.208355)

Morris MJ \& Chen H 2009 Established maternal obesity in the rat reprograms hypothalamic appetite regulators and leptin signaling at birth. International Journal of Obesity 33 115-122. (doi:10.1038/ijo.2008.213)

Muhlhausler B \& Smith SR 2009 Early-life origin of metabolic dysfunction: role of the adipocyte. Trends in Endocrinology and Metabolism 2 51-57. (doi:10.1016/j.tem.2008.10.006)

Muhlhausler BS, Adam CL, Findlay PA, Duffield JA \& McMillen IC 2006 Increased maternal nutrition alters development of the appetiteregulating network in the brain. FASEB Journal 20 1257-1259. (doi:10.1096/fj.05-5241fje)

Orozco-Solís R, Matos RJ, Lopes de Souza S, Grit I, Kaeffer B, Manhães de Castro R \& Bolaños-Jiménez F 2011 Perinatal nutrient restriction induces long-lasting alterations in the circadian expression pattern of genes regulating food intake and energy metabolism. International Journal of Obesity 7 990-1000. (doi:10.1038/ijo.2010.223) 
Ozanne SE, Dorling MW, Wang CL \& Nave BT 2001 Impaired PI3-kinase activation in adipocytes from early growth-restricted male rats. American Journal of Physiology. Endocrinology and Metabolism 3 E534-E539.

Palou M, Torrens JM, Priego T, Sánchez J, Palou A \& Picó C 2011 Moderate caloric restriction in lactating rats programs their offspring for a better response to HF diet feeding in a sex-dependent manner. Journal of Nutritional Biochemistry 6 574-584. (doi:10.1016/j.jnutbio.2010.05.001)

Patterson CM, Bouret SG, Park S, Irani BG, Dunn-Meynell AA \& Levin BE 2010 Large litter rearing enhances leptin sensitivity and protects selectively bred diet-induced obese rats from becoming obese. Endocrinology 151 4270-4279. (doi:10.1210/en.2010-0401)

Plagemann A, Harder T, Melchior K, Rake A, Rohde W \& Dörner G 1999a Elevation of hypothalamic neuropeptide Y-neurons in adult offspring of diabetic mother rats. Neuroreport 19 3211-3216. (doi:10.1097/ 00001756-199910190-00016)

Plagemann A, Harder T, Rake A, Waas T, Melchior K, Ziska T, Rohde W \& Dörner G 1999b Observations on the orexigenic hypothalamic neuropeptide Y-system in neonatally overfed weanling rats. Journal of Neuroendocrinology 11 541-546. (doi:10.1046/j.1365-2826.1999.00357.x)

Plagemann A, Harder T, Rake A, Janert U, Melchior K, Rohde W \& Dörner G 1999 c Morphological alterations of hypothalamic nuclei due to intrahypothalamic hyperinsulinism in newborn rats. International Journal of Developmental Neuroscience 17 37-44. (doi:10.1016/S07365748(98)00064-1)

Plagemann A, Harder T, Rake A, Melchior K, Rohde W \& Dorner G 2000 Hypothalamic nuclei are malformed in weanling offspring of low protein malnourished rat dams. Journal of Nutrition 130 2582-2589.

Plagemann A, Harder T, Brunn M, Harder A, Roepke K, Wittrock-Staar M, Ziska T, Schellong K, Rodekamp E, Melchior K et al. 2009 Hypothalamic proopiomelanocortin promoter methylation becomes altered by early overfeeding: an epigenetic model of obesity and the metabolic syndrome. Journal of Physiology 587 4963-4976. (doi:10.1113/jphysiol. 2009.176156)

Poulos SP, Hausman DB \& Hausman GJ 2010 The development and endocrine functions of adipose tissue. Molecular and Cellular Endocrinology 323 20-34. (doi:10.1016/j.mce.2009.12.011)

Rajia S, Chen H \& Morris MJ 2010 Maternal overnutrition impacts offspring adiposity and brain appetite markers-modulation by postweaning diet. Journal of Neuroendocrinology 22 905-914. (doi:10.1111/j.1365-2826. 2010.02005.x)
Ravelli AC, van Der Meulen JH, Osmond C, Barker DJ \& Bleker OP 1999 Obesity at the age of $50 \mathrm{y}$ in men and women exposed to famine prenatally. American Journal of Clinical Nutrition 70 811-816.

Samuelsson AM, Matthews PA, Argenton M, Christie MR, McConnell JM, Jansen EH, Piersma AH, Ozanne SE, Twinn DF, Remacle C et al. 2008 Diet-induced obesity in female mice leads to offspring hyperphagia, adiposity, hypertension, and insulin resistance: a novel murine model of developmental programming. Hypertension 51 383-392. (doi:10.1161/HYPERTENSIONAHA.107.101477)

Srinivasan M, Mitrani P, Sadhanandan G, Dodds C, Shbeir-ElDika S, Thamotharan S, Ghanim H, Dandona P, Devaskar SU \& Patel MS 2008 A high-carbohydrate diet in the immediate postnatal life of rats induces adaptations predisposing to adult-onset obesity. Journal of Endocrinology 197 565-574. (doi:10.1677/JOE-08-0021)

Steculorum SM \& Bouret SG 2011 Maternal diabetes compromises the organization of hypothalamic feeding circuits and impairs leptin sensitivity in offspring. Endocrinology 152 4171-4179. (doi:10.1210/en. 2011-1279)

Stevens A, Begum G, Cook A, Connor K, Rumball C, Oliver M, Challis J, Bloomfield F \& White A 2010 Epigenetic changes in the hypothalamic proopiomelanocortin and glucocorticoid receptor genes in the ovine fetus after periconceptional undernutrition. Endocrinology 151 3652-3664. (doi:10.1210/en.2010-0094)

Sutton GM, Centanni AV \& Butler AA 2010 Protein malnutrition during pregnancy in C57BL/6J mice results in offspring with altered circadian physiology before obesity. Endocrinology 4 1570-1580. (doi:10.1210/en. 2009-1133)

Wang P, Mariman E, Renes J \& Keijer J 2008 The secretory function of adipocytes in the physiology of white adipose tissue. Journal of Cellular Physiology 216 3-13. (doi:10.1002/jcp.21386)

Wright TM, Fone KC, Langley-Evans SC \& Voigt JP 2011 Exposure to maternal consumption of cafeteria diet during the lactation period programmes feeding behaviour in the rat. International Journal of Developmental Neuroscience 29 785-793. (doi:10.1016/j.ijdevneu.2011. 09.007)

Xiao XQ, Williams SM, Grayson BE, Glavas MM, Cowley MA, Smith MS \& Grove KL 2007 Excess weight gain during the early postnatal period is associated with permanent reprogramming of brown adipose tissue adaptive thermogenesis. Endocrinology 148 4150-4159. (doi:10.1210/ en.2007-0373)

Received in final form 17 September 2012

Accepted 29 October 2012

Accepted Preprint published online 29 October 2012 http://joe.endocrinology-journals.org DOI: 10.1530/JOE-12-0157
(C) 2013 Society for Endocrinology Printed in Great Britain
Published by Bioscientifica Ltd. 